\title{
Optical properties of chalcopyrite-type intermediate transition metal band materials from first principles
}

\author{
I. Aguilera , P. Palacios, P. Wahnón \\ Instituto de Energia Solar and Departamiento de Tecnologías Especiales, ETSI de Telecomunicación, UPM. Ciudad Universitaria s/n, Madrid, Spain
}

\begin{abstract}
The optical properties of a novel potential high-efficiency photovoltaic material have been studied. This material is based on a chalcopyritetype semiconductor $\left(\mathrm{CuGaS}_{2}\right)$ with some Ga atom substituted by Ti and is characterized by the formation of an isolated transition-metal band between the valence band and the conduction band. We present a study in which ab-initio density functional theory calculations within the generalized gradient approximation are carried out to determine the optical reflectivity and absorption coefficient of the materials of interest. Calculations for the host semiconductor are in good agreement with experimental results within the limitations of the approach. We find, as desired, that because of the intermediate band, the new Ti-substituted material would be able to absorb photons of energy lower than the band-gap of the host chalcopyrite. We also analyze the partial contributions to the main peaks of its spectrum.
\end{abstract}

(C) 2007 Elsevier B.V. All rights reserved.

Keywords: Chalcopyrite; Intermediate band materials; Optical properties

\section{Introduction}

The interest in chalcopyrite-type semiconductors has increased in recent years due to their technological applications. Of particular interest to us is the compound $\mathrm{CuGaS}_{2}$. Because its band-gap lies in the visible part of the spectrum, one of its more interesting applications is in photovoltaic devices. As a consequence of these characteristics, several experimental data are available for this material, allowing us to check the theoretical results of this work.

In a recent work, we proposed transition metal-substituted chalcopyrites $\left(\mathrm{Cu}_{4} \mathrm{MGa}_{3} \mathrm{~S}_{8}\right.$, with $\mathrm{M}=\mathrm{Ti}, \mathrm{V}, \mathrm{Cr}$ and $\left.\mathrm{Mn}\right)$ as candidates for presenting an isolated partially-filled narrow band between the valence band and the conduction band of the host semiconductor In practice, this transition metal band would allow this material to be used in highefficiency solar cells because of the absorption of sub-bandgap energy photons. The concept of an intermediate-band solar cell is based on this efficient use of the solar spectrum, which could increase the efficiency of the cell up to $63.1 \%$
More recently, calculations of the stability of this kind of material at different dilutions [4] have shown that when the transition metal is $\mathrm{Ti}$, it is of particular interest. Experimental preparations of these materials as thin films have already been initiated.

The main contribution of the present work is the detailed study of the optical reflectivity and absorption coefficient of Ti-substituted $\mathrm{CuGaS}_{2}$ as a promising highefficiency solar cell material. We will also identify the transitions responsible for the different structures in the optical conductivity.

All our calculations are based on density functional theory (DFT). In general, a high-quality description of the frequencydependent functions would require methods beyond DFT, however DFT can provide optical spectra in qualitative agreement with experiments. The purpose of this work is to confirm the effect of the enhancement in the optical properties of the material resulting from the intermediate transition metal band.

\section{Basic theory and method}

The crystal structure of our $\mathrm{Cu}_{4} \mathrm{TiGa}_{3} \mathrm{~S}_{8}$ model is generated by the vectors of the body-centred tetragonal 
$\mathrm{CuGaS}_{2}$ cell $(a, b, c)$, according to the equations $a^{\prime}=(a+b+$ c) $/ 2, b^{\prime}=(a+b+c) / 2, a^{\prime}=(b-a) \quad$ This tetragonal structure is responsible for the two independent components of the optical spectra, since the magnitude of the optical properties depends on the polarization of the photons. The first component corresponds to the polarizations along the $x$ and $y$ axes (labelled as $\varepsilon \perp$, it is the average of the $x$ and $y$ contributions) and the second one is due to the polarization in direction $z\left(\varepsilon_{\|}\right)$.

The electronic ground state of each material was calculated using DFT in the generalized gradient approximation (GGA), relaxing lattice parameters and ionic positions and allowing spin polarization. The tolerance for atomic forces in both relaxations was $0.01 \mathrm{eV} / \AA$. All our calculations were carried out with the plane-wave code VASP and using projector augmented wave (PAW) pseudopotentials

Furthermore, we need to obtain values for the two different components of the real and imaginary parts of the dielectric function. Therefore, it is necessary to obtain the components of the frequency-dependent dielectric tensor first, both the imaginary and the real part. The expression for the imaginary part can be written as the sum over occupied and unoccupied states in Eq. (1).

$$
\begin{aligned}
\varepsilon_{\alpha \beta}^{(2)}(\omega)= & \frac{4 \pi^{2} e^{2}}{\Omega m^{2} \omega^{2}} \sum_{k n n^{\prime} \sigma} f_{n \sigma}^{k}\left(1-f_{n^{\prime} \sigma}^{k}\right) \delta\left(\xi_{k n^{\prime}}-\xi_{k n}-\hbar \omega\right) \\
& \times\left\langle\psi_{n k \sigma}\left|p_{\alpha}\right| \psi_{n^{\prime} k \sigma}\right\rangle\left\langle\psi_{n k^{\prime} \sigma}\left|p_{\beta}\right| \psi_{n k \sigma}\right\rangle
\end{aligned}
$$

where functions $\psi_{n \mathbf{k} \sigma}$ and $\psi_{n / \mathrm{k} \sigma}$ are the crystal wavefunctions corresponding to the initial and final states at the k-point $\mathbf{k}$. We use the wavefunctions previously obtained in the ground state calculations. $e, m$, and $\Omega$ are the electron charge, its mass and the volume of the unit cell respectively. $f_{n \sigma}^{\mathrm{k}}$ is the Fermi distribution. To get a converged frequency-dependent dielectric tensor, the Brillouin zone has been sampled using $12 \times 12 \times 12$ Monkhorst-Pack grids including the gamma point. Within the notation noted above, we define $\varepsilon^{(2)}=$ $\left(\varepsilon \|^{2)}+2 \varepsilon\left(\mathcal{L}^{2}\right) / 3\right.$.

The real part of the dielectric tensor can be then obtained from the imaginary part by the Kramers-Krönig relations Eq. (2).

$\varepsilon_{\alpha \beta}^{(1)}(\omega)=1+\frac{2}{\pi} \wp \int_{0}^{\infty} \frac{\omega^{\prime}}{\omega^{\prime 2}-\omega^{2}+i \eta} \varepsilon_{\alpha \beta}^{(2)}\left(\omega^{\prime}\right) d \omega^{\prime}$

( $\wp$ indicates the principal value of the integral and $\eta$ is an infinitesimal number). Further details on this method can be found in references The integration range used to reach convergence in the dielectric function was $25 \mathrm{eV}$.

Once the dielectric function is obtained, many other interesting optical properties can be deduced. For photovoltaic purposes, we are particularly interested in absorption coefficients and reflectivities.

In this work, we only consider interband and direct transitions, and no local-field effects are taken into account. Since indirect transitions involve three particles, we may expect that the contribution of these transitions would be much lower than that of the direct ones.

\section{Results and discussions}

\subsection{Host $\mathrm{CuGaS_{2 }}$ semiconductor}

Full relaxations of the host $\mathrm{CuGaS}_{2}$ structures have been carried out previous to the study of the optical properties. A reasonable agreement with experimental results is obtained. The body-centred tetragonal structure of the chalcopyrite shows a lattice constant $a$ of $5.382 \AA$ and $c / a$ ratio of 1.981 . Reference

shows experimental values of $5.34 \AA$ and 1.98 , we also compare our results with those of Laksari their theoretical calculations (within the local density approximation LDA) lead to values of $5.263 \AA$ and 1.972. These differences are acceptable, using the GGA approach which, as is well known, overestimates the structural constants.

In order to get the best optical spectra within our approach, we need high-quality electronic properties. These electronic properties of both the host and the Ti-substituted chalcopyrite were obtained recently by our group.

In this respect, the calculated GGA band-gap is $0.68 \mathrm{eV}$ as compared to the experimental value of $2.43 \mathrm{eV}$ This is not surprising, since it is well known that the LDA and GGA are not suitable for treating excited states of a material and so they underestimate the value of the band-gap of the semiconductors. Our aim is to obtain the frequency-dependent dielectric function. However, both the real and the imaginary parts of the dielectric function have a high dependence on the value of the band-gap. To overcome this problem, a scissor operator is applied to adjust the band-gap from our calculation to match the experimental value.

The dielectric function determines the optical properties studied. In Fig. 1 we show the absorption coefficient and reflectivity of the $\mathrm{CuGaS}_{2}$ host semiconductor in both polarizations, compared with available experimental results determined by spectroscopic ellipsometry. Ab-initio calculations share a difficulty in predicting both the intensities and the peaks positions in the optical spectrum . Keeping this in mind, our results can be considered in good agreement with experimental measurements as well as with other theoretical results The main differences in the spectra concern the positions of the peaks as well as their intensities, whereas the general shape of the curves are very similar.

Regarding the reflectivity $(R)$, we know that a smaller energy band-gap yields a larger value of $\varepsilon^{(1)}(0)$, and thus a larger $R$. The use of the scissor operator partially solves this problem but as we can see in Fig. 1 even with the scissor correction, the calculated reflectivity is still higher than the experimental spectrum. This may be due to the neglect of the local-field effects, since local-field effects usually reduce the intensity of the peaks Besides, within our approach, some additional parameters should be considered as potential sources of error, as we are only taking into account direct and interband transitions.

Another interesting optical parameter is the refractive index at the zero frequency limit $n(0)$, and this property is also highly dependent on the value of the band-gap. In Table 1 we present the results of our calculations of $n(0)$ both with and without the 

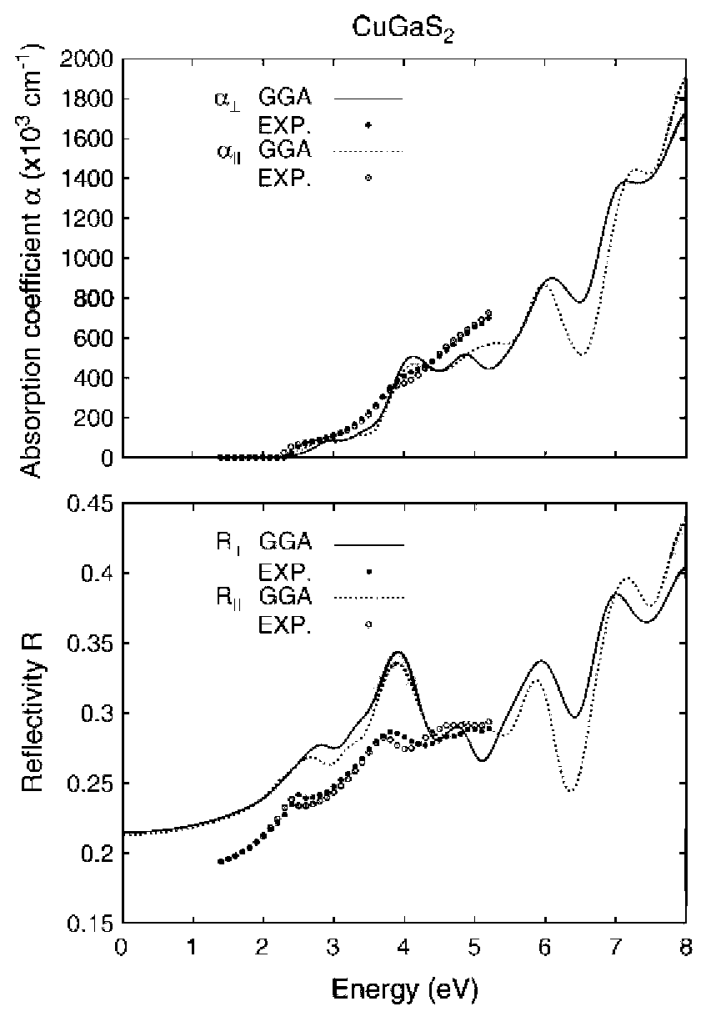

Fig. 1. Absorption coefficient and optical reflectivity of chalcopyrite $\mathrm{CuGaS}_{2}$ as calculated by the first principles DFT-GGA methods. Experimental measurements by Alonso A scissor operator is applied to make the theoretical band-gap match the experimental value. Symbols $\perp$ and $\|$ denote the two independent components of the functions.

scissor operator and compared with the experimental values stated by Boyd and theoretical results of Laksari

The values shown in this table are a clear justification of the use of the scissor correction.

These results presented for the $\mathrm{CuGaS}_{2}$ will be used to compare with the $\mathrm{Cu}_{4} \mathrm{TiGa}_{3} \mathrm{~S}_{8}$ spectra and to confirm the increase of the absorption of low-energy photon on which the intermediate-band concept is based.

\subsection{Ti-substituted $\mathrm{CuGaS}$}

The results of $\mathrm{Ti}$ substituting a $\mathrm{Ga}$ atom in the unit cell $\left(\mathrm{Cu}_{4} \mathrm{TiGa}_{3} \mathrm{~S}_{8}\right)$ were obtained using the same procedure. This alloy has a Ti atom dilution level of $12.5 \%$. After a structural optimization, a detailed study of the electronic properties was carried out and the results were compared with those of previous works. The absorption coefficient and reflectivity are

\section{Table 1}

Refractive index of the $\mathrm{CuGaS}_{2} n(0)$ and its two independent components: $n \perp(0)$ and $n_{\mid}(0)$, both with and without the scissor correction and compared with experimental and other theoretical results

\begin{tabular}{llll}
\hline & $n(0)$ & $n \perp(0)$ & $n_{\|}(0)$ \\
\hline GGA & 3.204 & 3.207 & 3.198 \\
GGA with corrections & 2.724 & 2.729 & 2.715 \\
Experimental [15] & 2.417 & 2.412 & 2.427 \\
Other work [9] & 2.62 & 2.611 & 2.623 \\
\hline
\end{tabular}
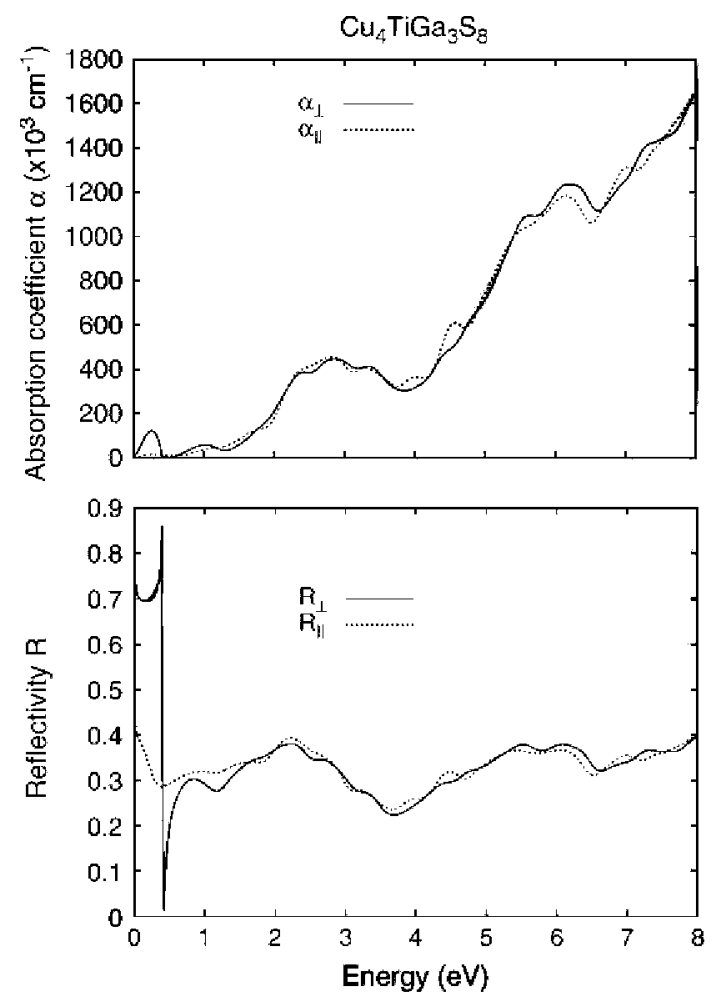

Fig. 2. Theoretical absorption coefficient and optical reflectivity of the intermediate-band material $\mathrm{Cu}_{4} \mathrm{TiGa}_{3} \mathrm{~S}_{8}$ in both components of the polarizations, $\perp$ and $\|$.

obtained and presented in Fig. 2, in both kinds of polarization. In the present case, the anisotropy is less pronounced than in the case of the host chalcopyrite. This happens because of the slightly different structure of both compounds. In some cases, symmetry forbids transitions in one polarization, for example we can see that the first peak in the absorption spectrum in Fig. 2 is only due to the perpendicular polarization. This first peak can be attributed to transitions inside the intermediate band as we will see below. These transitions are also responsible for the low-energy fluctuations in the reflectivity spectrum, because of the metallic character of the intermediate band.

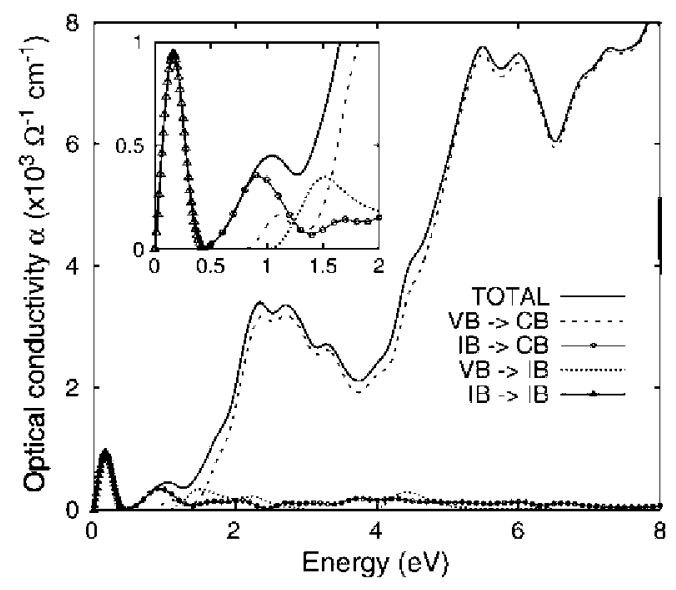

Fig. 3. Partial contributions of the four different kinds of transitions to the total optical conductivity of $\mathrm{Cu}_{4} \mathrm{TiGa}_{3} \mathrm{~S}_{8}$. 

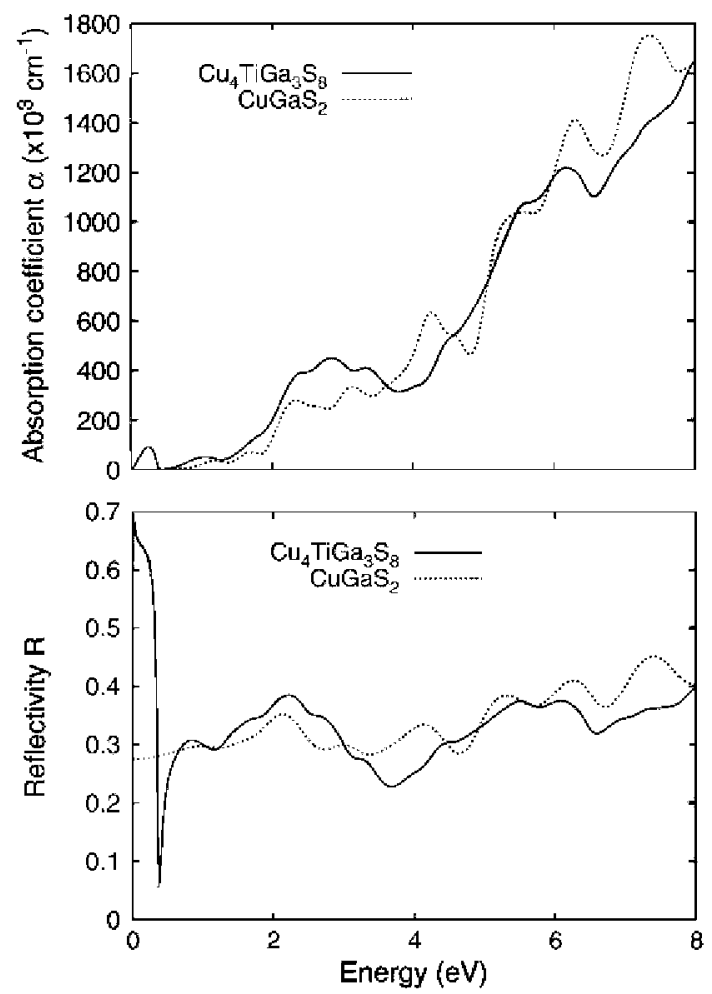

Fig. 4. Absorption coefficient and optical reflectivity of the intermediate-band material $\mathrm{Cu}_{4} \mathrm{TiGa}_{3} \mathrm{~S}_{8}$ compared with those of the host $\mathrm{CuGaS}_{2}$. In this case we represent the average of the three components of the polarization. No scissor correction is applied.

For photovoltaic purposes, it is very interesting to determine which transitions contribute to each peak in the spectra; this allows us to understand the effect of the intermediate band in the absorption. We have made a detailed analysis of these partial contributions by means of the optical conductivity $\sigma$, which relates to the imaginary part of the dielectric function according to $\sigma \propto \omega \varepsilon^{(2)}$. Fig. 3 shows partial conductivities related to direct transitions being: from the valence band (VB) to the conduction band (CB), which are responsible for the main contribution; from the $V B$ to the intermediate band (IB), with onset energy at about $1 \mathrm{eV}$; from the IB to the $\mathrm{CB}$, which begins at energy $0.4 \mathrm{eV}$; and finally transitions inside the intermediate band, the first peak in the energy range $0-0.4 \mathrm{eV}$. This figure helps in the understanding of the effect of the intermediate band since it shows the expected increase in the absorption of low-energy photons and that the enhancement is produced because of the transitions involving the IB.

\subsection{Comparison between $\mathrm{Cu}_{4} \mathrm{TiGa}_{3} \mathrm{~S}_{8}$ and $\mathrm{CuGaS_{2 }}$}

The effect on the optical properties of the Ti inclusion in the chalcopyrite semiconductor can also be studied by comparison of the $\mathrm{Cu}_{4} \mathrm{TiGa}_{3} \mathrm{~S}_{8}$ spectra with that of the $\mathrm{CuGaS}_{2}$. In Fig. 4 we present the average of the two components of the absorption coefficient and reflectivity of both compounds. In this case, no scissor operator is applied. As we can see, the absorption of the Tisubstituted alloy begins at almost zero energy, as a consequence of transitions inside the intermediate band, while the spectrum of the host chalcopyrite begins at the theoretical value of the band-gap. Even though the overall absorption spectrum of $\mathrm{Cu}_{4} \mathrm{TiGa}_{3} \mathrm{~S}_{8}$ is no greater than that of the $\mathrm{CuGaS}_{2}$, we get a significantly higher absorption for energies lower than about $4 \mathrm{eV}$. Bearing in mind that the most intense part of the sun's emissions are in the energy range of $0-5 \mathrm{eV}$, this gain in the absorption should be considered as a promising result. This is important, since it is the aim of the intermediate-band solar cell concept.

\section{Conclusions}

In summary, we have presented results concerning ab-initio properties and optical spectra for two interesting photovoltaic materials: the semiconductor $\mathrm{CuGaS}_{2}$ and the intermediate transition metal band material $\mathrm{Cu}_{4} \mathrm{TiGa}_{3} \mathrm{~S}_{8}$. The latter presents the desired enhancement of the absorption spectrum thanks to the use of low-energy photons on which the intermediate-band solar cell concept is based. We have also found the dependence of the optical properties on the polarization of the photons.

In this work only interband and direct transitions are considered. Since we have carried out calculations on the phonon spectra of these materials we will be able to estimate the contribution of indirect transitions in further work. But as indirect transitions involve three particles, the contribution of these transitions is expected to be much lower than that of the direct ones. In any case, we expect to go further in our studies and carry out higher accuracy calculations of the optical properties.

A partial study of the optical conductivity peaks has been done in the case of the intermediate-band material $\mathrm{Cu}_{4} \mathrm{TiGa}_{3} \mathrm{~S}_{8}$ to understand the overall contribution of the intermediate band to the total optical properties. We see that the electronic structure of this material allows the absorption of low-energy photons that is forbidden in the host semiconductor, thus increasing the absorption coefficient in the main region of the solar spectrum.

All the properties studied would be more valuable in comparison with experimental results. We believe that this work will help in the understanding of future experiments and we expect to have experimental results available soon.

\section{References}

P. Palacios, K. Sánchez, J.C. Conesa, P. Wahnón, Phys. Status Solidi (a) 203 (No. 6) (2006) 1395.

P. Palacios, K. Sánchez, J.C. Conesa, J.J. Fernández, P. Wahnón, Thin Solid Films 515 (2007) 6280. 
A. Luque, A. Martí, Phys. Rev. Lett. 78 (1997) 5014.

P. Palacios, K. Sánchez, P. Wahnón, J.C. Conesa, J. Solar Energy Eng. 129 (2007) 314.

G. Kresse, J. Hafner, Phys. Rev., B 47 (1993) RC558;

G. Kresse, J. Furthmüller, Phys. Rev., B 54 (1996) 11169;

G. Kresse, J. Joubert, Phys. Rev., B 59 (1999) 1758.

B. Adolph, J. Furthmüller, F. Bechstedt, Phys. Rev., B 63 (2001) 125108

M. Gajdos, K. Hummer, G. Kresse, J. Furthmüller, F. Bechstedt, Phys. Rev., B 73 (2006) 045112.

S.C. Abraham, J.L. Bernstein, J. Chem. Phys. 59 (1973) 4515.

S. Laksari, A. Chahed, N. Abbouni, O. Benhelal, B. Abbar, Comput. Mater. Sci. 38 (2006) 223.
J.L. Shay, J.H. Wernick, Ternary Chalcopyrite Semiconductors: Growth Electronic Properties and Applications, Pergamon Press, Oxford, 1974. M.I. Alonso, K. Wakita, J. Pascual, M. Garriga, N. Yamamoto, Phys. Rev., B 63 (2001) 075203 .

James L.P. Hughes, J.E. Sipe, Phys. Rev., B 53 (1996) 10751.

R. Ahuja, S. Auluck, O. Eriksson, J.M. Wills, B. Johansson, Sol. Energy Mater. Sol. Cells 53 (1998) 357.

S. Lebègue, B. Arnaud, M. Alouani, Phys. Rev., B 72 (2005) 085103. G.D. Boyd, H. Kasper, J.H. McFee, IEEE J. Quantum Electron. QE-7 (1971) 563.

P. Palacios, I. Aguilera, P. Walnón, Thin Solid Films 516 (2008) 7070, doi:10.1016/j.tsf.2007.12.062. 